\title{
Dapivirine Vaginal Ring Use Does Not Diminish the Effectiveness of Hormonal Contraception
}

\author{
Jennifer E. Balkus, PhD, MPH, *† Thesla Palanee-Phillips, PhD, $\S$ Krishnaveni Reddy, MBChB, $\S$ \\ Samantha Siva, MBChB, || Ishana Harkoo, MBChB, ף Clemensia Nakabiito, MBChB,\# \\ Kenneth Kintu, MBChB,\# Gonasangrie Nair, MBChB, ** Catherine Chappell, MD, $\dagger$ \\ Flavia Matovu Kiweewa, MBChB,\# Samuel Kabwigu, MBChB,\# Logashvari Naidoo, MBChB,\| \\ Nitesha Jeenarain, MBChB, \| Mark Marzinke, PhD, $\neq \neq$ Lydia Soto-Torres, MD, $\S \S$ \\ Elizabeth R. Brown, ScD, *\|\| and Jared M. Baeten, MD, PhD, $+\$ \mathbb{\|}$ on behalf of the MTN-020/ASPIRE \\ Study Team
}

\begin{abstract}
Objective: To evaluate the potential for a clinically relevant drugdrug interaction with concomitant use of a dapivirine vaginal ring, a novel antiretroviral-based HIV-1 prevention strategy, and hormonal contraception by examining contraceptive efficacies with and without dapivirine ring use.
\end{abstract}

Design: A secondary analysis of women participating in MTN-020/ ASPIRE, a randomized, double-blind, placebo-controlled trial of the dapivirine vaginal ring for HIV-1 prevention.

Methods: Use of a highly effective method of contraception was an eligibility criterion for study participation. Urine pregnancy tests

Received for publication January 13, 2017; accepted April 17, 2017.

From the *Vaccine and Infectious Disease Division, Fred Hutchinson Cancer Research Center, Seattle, WA; Departments of †Epidemiology; †Global Health, University of Washington, Seattle, WA; \$Wits Reproductive Health and HIV Institute, Johannesburg, South Africa; |South African Medical Research Council, Durban, South Africa; 9 CAPRISA, Durban, South Africa; \#Makerere University - Johns Hopkins University Research Collaboration, Kampala, Uganda; **Emavundleni Research Centre, Cape Town, South Africa; ††Magee-Womens Hospital of UPMC, Pittsburgh, PA; + Departments of Pathology and Medicine, Johns Hopkins University School of Medicine, Baltimore, MD; $\S \S U$ National Institutes of Health, Bethesda, MD; and Departments of $\|||$ Biostatistics; q9Medicine, University of Washington, Seattle, WA

The Microbicide Trials Network (MTN) is funded by National Institute of Allergy and Infectious Disease (UM1AI068633, UM1AI068615, and UM1AI106707), with cofunding from the Eunice Kennedy Shriver National Institute of Child Health and Human Development and National Institute of Mental Health, all components of the US National Institutes of Health (NIH). The content is solely the responsibility of the authors and does not necessarily represent the official views of the NIH.

Presented in part at the Conference on Retroviruses and Opportunistic Infections; February 13-17, 2017; Seattle, WA.

The authors have no conflicts of interest to disclose.

J.E.B. and J.M.B. conceptualized the article and analysis plan. J.E.B. conducted the analysis in collaboration with J.M.B. T.P.P., K.R., S.S., I.H., C.N., K.K., G.N., F.M.K., S.K., L.N., N.J., and L.S.T. contributed to data collection. M.M. generated data on dapivirine detection in plasma. J.E.B. drafted the initial report and all authors contributed to article content and approved the final manuscript.

Correspondence to: Jennifer E. Balkus, PhD, MPH, Fred Hutchinson Cancer Research Center, 1100 Fairview Avenue N., M2-C200, PO Box 19024, Seattle, WA 98109-1024 (e-mail: jbalkus@fredhutch.org).

Copyright () 2017 Wolters Kluwer Health, Inc. All rights reserved. were performed monthly. Pregnancy incidence by arm was calculated separately for each hormonal contraceptive method and compared using an Andersen-Gill proportional hazards model stratified by site and censored at HIV-1 infection.

Results: Of 2629 women enrolled, 2310 women returned for follow-up and reported using a hormonal contraceptive method at any point during study participation (1139 in the dapivirine arm and 1171 in the placebo arm). Pregnancy incidence in the dapivirine arm versus placebo among women using injectable depot medroxyprogesterone acetate was $0.43 \%$ vs. $0.54 \%$, among women using injectable norethisterone enanthate was $1.15 \%$ vs. $0 \%$, among women using hormonal implants was $0.22 \%$ vs. $0.69 \%$, and among women using oral contraceptive pills was $32.26 \%$ vs. $28.01 \%$. Pregnancy incidence did not differ by study arm for any of the hormonal contraceptive methods.

Conclusions: Use of the dapivirine ring does not reduce the effectiveness of hormonal contraceptives for pregnancy prevention. Oral contraceptive pill use was associated with high pregnancy incidence, potentially because of poor pill adherence. Injectable and implantable methods were highly effective in preventing pregnancy.

Key Words: dapivirine, NNRTI, hormonal contraception, pregnancy (J Acquir Immune Defic Syndr 2017;76:e47-e51)

\section{INTRODUCTION}

Prevention of HIV-1 and unplanned pregnancy are global public health priorities for reproductive-aged women. ${ }^{1}$ For women with or at risk of HIV-1, concurrent use of hormonal contraceptives for pregnancy prevention and antiretrovirals for HIV-1 treatment or prevention could result in potential drug-drug interactions that may diminish contraceptive effectiveness. ${ }^{2,3}$ Contraceptive hormones are metabolized by hepatic cytochrome P450 enzymes, and certain non-nucleoside reverse transcriptase inhibitors (NNRTIs) can induce cytochrome P450 enzymes leading to reduced plasma progestin concentrations, with some NNRTIs having a greater impact on progestin pharmacokinetics than others. ${ }^{4-8}$ Among HIV-1-infected women, use of the NNRTI efavirenz as part 
of combination HIV-1 treatment has been associated with a reduced effectiveness of contraceptive implants. ${ }^{3,6,7,9}$

A vaginal ring containing dapivirine, a novel NNRTI, demonstrated effectiveness for HIV-1 prevention, ${ }^{10,11}$ with HIV-1 incidence reduced in 2 randomized trials by approximately one-third overall and by $>50 \%$ among those with biologic evidence of ring use. ${ }^{11,12}$ Pregnancy incidence and outcomes did not differ between those receiving active dapivirine vaginal ring and placebo ${ }^{13}$; however, the potential for a clinically relevant drug-drug interaction between vaginally delivered dapivirine and hormonal contraceptive effectiveness has not been assessed in epidemiologic studies. Among women who participated in a phase III trial of the dapivirine vaginal ring, we compared the incidence of pregnancy in women randomized with the dapivirine ring versus placebo, stratified by hormonal contraceptive method.

\section{METHODS}

This is a secondary analysis of data from MTN-020/ ASPIRE, a randomized, double-blind, placebo-controlled phase III safety and effectiveness study of the dapivirine vaginal ring for HIV-1 prevention (Clinicaltrials.gov NCT01617096). Detailed methods and results for the trial have been published. ${ }^{11}$ Briefly, between August 2012 and June 2014, 2629 healthy, sexually active, HIV-1-uninfected women aged 18-45 years from Malawi, South Africa, Uganda, and Zimbabwe were enrolled. Women who were pregnant or intending to become pregnant were not eligible for enrollment and use of a highly effective method of contraception was a trial eligibility criterion. Eligible women were randomly assigned in equal proportions to receive either the dapivirine vaginal ring or placebo ring. Participants provided written informed consent, and applicable local and national ethical and regulatory authorities approved the study protocol.

At enrollment and monthly follow-up visits, standardized face-to-face interviews were conducted to collect data on the contraceptive method used since the participant's last visit, as well as reported condom use and sexual behaviors. Contraceptive methods were provided on-site or could be obtained from local health providers and women were permitted to change contraceptive method during follow-up. Urine pregnancy tests and HIV-1 rapid antibody tests were performed monthly. If a participant became pregnant, study product was withheld for the duration of pregnancy and breastfeeding and the participant was referred for pregnancy management or antenatal care. Study product was permanently discontinued after HIV-1 seroconversion. To objectively monitor adherence to study product during the trial, plasma samples were collected quarterly and were tested for dapivirine using a validated liquid chromatography-mass spectrometry assay. ${ }^{11,14}$

Pregnancy incidence by arm was calculated separately for each hormonal contraceptive method [injectable depot medroxyprogesterone acetate (DMPA), injectable norethisterone enanthate (NET-EN)], hormonal implants, and oral contraceptive pills (OCPs). The pregnancy incidence with typical use of injectables (DMPA and NET-EN), OCPs, and

e48 | www.jaids.com hormonal implants is estimated to be: 6,9 , and $<1$ per 100 person-years, respectively. ${ }^{15}$ The follow-up time when participants reported nonhormonal contraceptive methods [eg, intrauterine device and tubal ligation] or no method were excluded from analysis. Incident pregnancy was defined as the first visit with a positive urine pregnancy test. Visits while pregnant were censored and participants re-entered the risk set after completion of the pregnancy. Visits after HIV-1 seroconversion were also censored. For strata with no observed pregnancies, the $95 \%$ confidence interval (CI) for the incidence was calculated using Hanley approach for calculating CIs when no events are observed and Miettinen modification for the exact test to calculate the upper bound of the CI. ${ }^{16,17}$

Pregnancy incidence by hormonal contraceptive method was first compared using univariate Andersen-Gill proportional hazards models stratified by site, which allows for inclusion of recurrent events ${ }^{18}$; contraceptive method was included as a time-dependent variable. Multivariable models were performed that included the following factors selected a priori: age, marital status, number of live births at enrollment, and reported condom use at last vaginal sex act (time-varying factor). Additional factors were considered for inclusion in the multivariable model if they differed by study arm and were associated with incident pregnancy $(P<0.1)$. A sensitivity analysis was performed categorizing participants in the dapivirine arm based on plasma concentrations of dapivirine from the quarterly visits. Dapivirine plasma concentrations for intervening visits were imputed using last value carried forward. Participants with plasma dapivirine levels $>95 \mathrm{pg} / \mathrm{mL}$ were considered to have had recently used the ring (a level which corresponds to $\geq 8$ hours of continuous ring use). ${ }^{11,14}$ Pregnancy incidence among participants with recent ring use was compared with participants with no recent ring use $(\leq 95 \mathrm{pg} / \mathrm{mL}$ dapivirine detected in plasma) and participants in the placebo arm using the same methods described above. Analyses were performed using Stata, version 14.0 (StataCorp., College Station, TX).

\section{RESULTS}

Of 2629 women enrolled, 3 were found to be HIV-1 infected at enrollment, 12 never returned for follow-up, 78 had a tubal ligation, and 226 used a copper intrauterine devicethroughout study participation, leaving 2310 women who used a hormonal contraceptive method during follow-up (1139 in the dapivirine arm and 1171 in the placebo arm) (Table 1). Overall, 55\% of participants were $<27$ years of age, $38 \%$ were married, and $54 \%$ reported 2 or more live births. The most commonly reported method of hormonal contraception was injectable depot medroxyprogesterone acetate [DMPA] $(46 \%$ of participants reported use at enrollment; DMPA use comprised $45 \%$ of the total followup time included in this analysis), followed by hormonal implants (22\% at enrollment; $28 \%$ of follow-up time), injectable norethisterone enanthate [NET-EN] (16\% at enrollment; $16 \%$ of follow-up time), and OCPs (12\% at enrollment; $11 \%$ of follow-up time). 
TABLE 1. Participant Characteristics*

\begin{tabular}{|c|c|c|}
\hline & $\begin{array}{c}\text { Dapivirine, } \\
\mathbf{N}=1139\end{array}$ & $\begin{array}{l}\text { Placebo, } \\
\mathrm{N}=1171\end{array}$ \\
\hline \multicolumn{3}{|l|}{ Age, yrs } \\
\hline $18-21$ & $253(21.2)$ & $236(20.2)$ \\
\hline $22-26$ & $365(32.1)$ & $414(35.4)$ \\
\hline $27-45$ & $521(45.7)$ & $521(44.5)$ \\
\hline Currently married $\dagger$ & 417 (36.6) & $463(39.5)$ \\
\hline \multicolumn{3}{|l|}{ Education } \\
\hline No schooling or primary only & $170(14.9)$ & $158(13.5)$ \\
\hline Some secondary education or more & $969(85.1)$ & $1013(86.5)$ \\
\hline Participant earns own income & $513(45.0)$ & $506(43.2)$ \\
\hline \multicolumn{3}{|l|}{ Number of live births } \\
\hline None & $121(10.6)$ & $133(11.4)$ \\
\hline 1 birth & $406(35.7)$ & $400(34.2)$ \\
\hline 2 or more & $612(53.7)$ & $638(54.5)$ \\
\hline Condom use at last vaginal sex act $+\underset{\dagger}{\dagger}$ & $680(59.7)$ & $665(56.8)$ \\
\hline More than 1 sex partner in the past $3 \mathrm{mo}$. & $182(16.0)$ & $192(16.4)$ \\
\hline Curable STI§ & $253(22.2)$ & $241(20.6)$ \\
\hline \multicolumn{3}{|l|}{ Contraceptive method at enrollment } \\
\hline DMPA & $512(45.0)$ & $554(47.3)$ \\
\hline NET-EN & $197(17.3)$ & $178(15.2)$ \\
\hline IUD\| & $38(3.3)$ & $59(5.0)$ \\
\hline Implant & $256(22.5)$ & $240(20.5)$ \\
\hline OCPs & $136(11.9)$ & $140(12.0)$ \\
\hline \multicolumn{3}{|l|}{ Contraceptive method follow-up time } \\
\hline DMPA & $697(43.8)$ & $746(46.3)$ \\
\hline NET-EN & $260(16.4)$ & $259(16.1)$ \\
\hline Implant & $458(28.8)$ & $435(27.0)$ \\
\hline OCPs & $174(11.0)$ & $171(10.6)$ \\
\hline
\end{tabular}

*Data presented as N (\%).

$\dagger$ Two participants in the placebo arm missing marital status.

†Use of male or female condoms at the last vaginal sex act.

$\S$ Defined as detection of $C$. trachomatis, $N$. gonorrhoeae, $T$. vaginalis, or syphilis at baseline. Two participants in the placebo arm and 1 participant in the dapivirine arm missing $T$. vaginalis results at baseline.

$\|$ Participants used an IUD at enrollment, but switched to hormonal method during follow-up. Participants who used an IUD throughout study participation were excluded from the analysis.

TPercentage calculated based on 3200 person-years of person time included in the analysis, 1589 person-years in the dapivirine arm, and 1611 person-years in the placebo arm.

IUD, intrauterine device; STI; sexually transmitted infection.

A total of 117 pregnancies occurred among 114 participants during use of a hormonal contraceptive method (63 pregnancies in the dapivirine ring arm and 54 in the placebo arm). Seven pregnancies occurred among women who reported using DMPA (incidence $=0.49$ per 100 personyears), 3 among women using NET-EN (incidence $=0.58$ per 100 person-years), 3 among women using hormonal implants (incidence $=0.45$ per 100 person-years), and 104 among women using OCPs (incidence $=30.15$ per 100 personyears). Pregnancy incidence did not differ for those randomized to the dapivirine vaginal ring versus placebo ring for any of the hormonal contraceptive methods in univariate or multivariable models (Table 2). Results were generally similar to sensitivity analyses that characterized participants in the dapivirine ring arm by dapivirine plasma concentrations; however, for women reporting DMPA, NET-EN, and
OCP use, pregnancy incidence was slightly higher among participants who had not recently used the dapivirine ring compared with those who appeared to be using the ring (Table 2).

\section{DISCUSSION}

In this secondary analysis of women participating in a randomized trial of the dapivirine vaginal ring for HIV-1 prevention, dapivirine ring use was not associated with diminished hormonal contraceptive effectiveness. These data provide important evidence in support of the dapivirine ring for HIV-1 prevention among contracepting women and add to the larger body of evidence that supports the use of antiretroviral-based HIV-1-prevention interventions among reproductive-aged women. Although dapivirine is metabolized by cytochrome P450 enzymes, it does not appear that dapivirine metabolism induces isoenzymes that are integral for hormone metabolism. ${ }^{19}$ Dapivirine did not induce CYP1A2 or CYP3A4/5 activity in cultured human hepatocytes at concentrations up to $100 \mathrm{ng} / \mathrm{mL}$, which is much higher than those observed in the plasma of women using the dapivirine ring [personal communication-Jeremy Nuttal, IPM]. Therefore, there is little potential for significant interactions with hormonal contraceptives. Furthermore, uptake of dapivirine occurs through topical absorption of drug eluted from the vaginal ring, which results in low systemic dapivirine concentrations, thus reducing the likelihood of a potential clinically relevant drug-drug interaction..$^{20,21}$

Although pregnancy incidence was similar by study arm within each hormonal contraceptive method, pregnancy incidence differed substantially across contraceptive methods. Injectable and implantable methods were highly effective in preventing pregnancy; however, OCP users had a high pregnancy incidence ( $\sim 30$ per 100 person-years). Although typical OCP use is reported to have a pregnancy incidence of approximately 9 per 100 person-years, ${ }^{15}$ other HIV-1prevention studies have seen substantially higher pregnancy rates in OCP users. ${ }^{22-24}$ Adherence to daily oral pills may be challenging for some women and could explain high pregnancy incidence for OCP users. Additionally, it has been hypothesized that some women in HIV-1-prevention trials may switch to OCPs as their contraceptive choice with the intention of becoming pregnant, because they could report OCP use but not necessarily use the method. Unfortunately, data on adherence to contraception and pregnancy intention during trial participation were not collected, so these hypotheses cannot be explored further.

In sensitivity analyses, where participants randomized to the dapivirine ring were characterized based on dapivirine plasma levels, we observed slightly higher pregnancy incidences among women with no recent ring use $(\leq 95 \mathrm{pg} / \mathrm{mL})$ compared with women with recent ring use for those using DMPA, NET-EN, or OCPs. These data suggest a correlation between adherence to user-dependent contraceptive methods and adherence to the study intervention, although these differences were not statistically significant and should be interpreted with caution because of the small number of pregnancies that occurred among DMPA and NET-EN users. 
TABLE 2. Pregnancy Incidence by Study Arm Stratified by Hormonal Contraceptive Method

\begin{tabular}{|c|c|c|c|c|c|c|}
\hline Method* & \multicolumn{2}{|r|}{ Study Arm } & \multicolumn{2}{|c|}{ \# Of Pregnancies } & \multicolumn{2}{|r|}{ \# Of Person-years } \\
\hline \multicolumn{7}{|c|}{ By randomization arm } \\
\hline \multirow[t]{2}{*}{ DMPA } & & Dapivirine & & 3 & & 697 \\
\hline & & Placebo & & 4 & & 746 \\
\hline NET-EN & & Placebo & & 0 & & 259 \\
\hline \multirow[t]{2}{*}{ OCPs } & & Dapivirine & & 56 & & 174 \\
\hline & & Placebo & & 48 & & 171 \\
\hline Implant & & Placebo & & 3 & & 435 \\
\hline \multicolumn{7}{|c|}{ By plasma drug detection\| } \\
\hline \multirow[t]{3}{*}{ DMPA } & & Dapivirine $>95 \mathrm{pg} / \mathrm{mL}$ & & 0 & & 509 \\
\hline & & Dapivirine $\leq 95 \mathrm{pg} / \mathrm{mL}$ & & 3 & & 109 \\
\hline & & Placebo & & 4 & & 747 \\
\hline NET-EN & & Dapivirine $>95 \mathrm{pg} / \mathrm{mL}$ & & 1 & & 178 \\
\hline OCPs & & Placebo & & 48 & & 171 \\
\hline \multirow[t]{3}{*}{ Implant } & & Dapivirine $>95 \mathrm{pg} / \mathrm{mL}$ & & 1 & & 378 \\
\hline & & Dapivirine $\leq 95 \mathrm{pg} / \mathrm{mL}$ & & 0 & & 40 \\
\hline & & Placebo & & 3 & & 435 \\
\hline Method* & Pregnar & cidence $\dagger(95 \%$ CI $)$ & Unadjusted & (95\% CI) & Adju & ed HR§ $(95 \% \mathrm{CI})$ \\
\hline \multicolumn{7}{|c|}{ By randomization arm } \\
\hline \multirow[t]{2}{*}{ DMPA } & 0.43 & $0.14-1.34$ & 0.78 & $0.17-3.52$ & 0.76 & $0.16-3.52$ \\
\hline & 0.54 & $0.20-1.43$ & Ref. & & Ref. & \\
\hline \multirow[t]{2}{*}{ NET-EN } & 1.15 & $0.37-3.58$ & Unable to calculate & Unable to calculate & & \\
\hline & 0 & $0.0-3.39$ & Ref. & & Ref. & \\
\hline \multirow[t]{3}{*}{ NET-EN } & 0.56 & $0.08-3.99$ & Unable to calculate & Unable to calculate & & \\
\hline & 1.91 & $0.27-13.56$ & Unable to calculate & Unable to calculate & & \\
\hline & 0 & $0.00-3.15$ & Ref. & & Ref. & \\
\hline \multirow[t]{3}{*}{ OCPs } & 27.71 & $19.49-39.40$ & 0.92 & $0.56-1.50$ & 1.07 & $0.65-1.76$ \\
\hline & 50.12 & $32.68-76.86$ & 1.71 & $0.99-2.95$ & 1.74 & $0.99-3.06$ \\
\hline & 28.01 & $21.11-37.17$ & Ref. & & Ref. & \\
\hline Implant & 0.26 & $0.04-1.88$ & 0.37 & $0.04-3.53$ & 0.33 & $0.03-3.24$ \\
\hline & 0 & $0.00-20.41$ & Unable to calculate & Unable to calculate & & \\
\hline & 0.69 & $0.22-2.14$ & Ref. & & Ref. & \\
\hline
\end{tabular}

*Women could report more than 1 option; therefore, an individual woman could contribute person-time to more than 1 method in this table. However, this was rare and occurred at 1 visit where a pregnancy was detected (OCP and implant use were reported for 1 participant in the placebo arm) and $<1 \%$ of visits without a pregnancy detected. Contraceptive method was considered a time-varying factor. For missed visits, contraceptive method was imputed using last value carried forward.

†Incidence presented per 100 person-years. For strata with no observed pregnancies, the $95 \%$ CI for the incidence was calculated using Hanley approach for calculating confidence intervals when no events are observed and Miettinen modification for the exact test. ${ }^{15,16}$

$\$$ Andersen-Gill proportional hazards models to allow for multiple pregnancies were used to evaluate the association between study arm and incident pregnancies among participants who reported using the method of interest. Only visits where that method was reported are included in the model. Unadjusted models are stratified by site.

§Andersen-Gill proportional hazards models to allow for multiple pregnancies were used to evaluate the association between study arm and incident pregnancies among participants who reported using the method of interest. Only visits where that method was reported are included in the model. Multivariable models are stratified by site and adjusted for the following factors: age category at enrollment, marital status at enrollment, number of live births reported at enrollment (category), and time-varying condom use at last vaginal sex act.

$\|$ No data were available for the first 2 follow-up visits (month 1 and month 2) since plasma dapivirine testing did not begin until month 3 ; therefore, pregnancies and person-time that occurred during this interval were excluded from this analysis, including 4 pregnancies among participants using OCPs and 1 in a participant using NET-EN. 
Among women participating in trials of oral preexposure prophylaxis (PrEP) with tenofovir-based pills for HIV-1 prevention, hormonal contraceptive plasma levels and contraceptive effectiveness were similar among women using PrEP compared with placebo. ${ }^{24,25}$ Our data add to the body of evidence supporting the use of antiretroviral-based PrEP for HIV-1 prevention among hormonal contraceptive users. However, as new antiretroviral-based HIV-1-prevention interventions are evaluated, it will be important to continue to assess the effect of these novel approaches on hormonal contraceptive effectiveness to ensure that women do not experience reduced protection against unintended pregnancy.

Our study has several strengths, including data collected as part of a multicountry phase III clinical trial with high retention, good levels of drug detection indicating recent ring use and monthly assessments of family planning method and pregnancy. Nonetheless, our findings should be interpreted in the context of limitations. There were few pregnancies among women who reported using implant and injectable methods; however, the pregnancy incidence overall and by arm was consistent with or below what would be expected with typical use for these methods. ${ }^{15}$ Contraceptive implants have had seemingly diminished effectiveness with concurrent use of the NNRTI efavirenz, but the pregnancy incidence among implant users in this study was lower than that observed in those studies $6,24,26$ including among women receiving the active dapirivine ring. Results were similar to our sensitivity analysis based on dapivirine plasma concentrations; however, plasma concentrations were only assessed during quarterly visits, whereas pregnancy was assessed monthly, which may result in misclassification if dapivirine ring use was not consistent at intervening visits.

In summary, our findings suggest that the dapivirine vaginal ring can be used in combination with a range of hormonal contraceptive methods without diminishing the effectiveness of contraception. These data support the use of the dapivirine vaginal ring for HIV-1 prevention among contracepting women and are important to consider as efforts to develop a coformulated vaginal ring for protection against HIV-1 acquisition and unintended pregnancy are underway.

\section{ACKNOWLEDGMENTS}

The authors gratefully acknowledge the contributions of the women who participated in ASPIRE/MTN-020. The authors express their sincere appreciation to the study team for their dedicated work on data and sample collection and to the MTN Statistical and Data Management Center for their work on data management.

\section{REFERENCES}

1. UNAIDS. Global plan towards the elimination of new HIV infections among children by 2015 and keeping their mothers alive. 2011. Available at: http://www.unaids.org/sites/default/files/media_asset/20110609_ JC2137_Global-Plan-Elimination-HIV-Children_en_1.pdf. Accessed October 12, 2016.

2. Polis CB, Phillips SJ, Hillier SL, et al. Levonorgestrel in contraceptives and multipurpose prevention technologies: does this progestin increase HIV risk or interact with antiretrovirals? AIDS. 2016;30:2571-2576.
3. Scarsi KK, Darin KM, Chappell CA, et al. Drug-drug interactions, effectiveness, and safety of hormonal contraceptives in women living with HIV. Drug Saf. 2016;39:1053-1072.

4. Vieira CS, Bahamondes MV, de Souza RM, et al. Effect of antiretroviral therapy including lopinavir/ritonavir or efavirenz on etonogestrel-releasing implant pharmacokinetics in HIV-positive women. J Acquir Immune Defic Syndr. 2014;66:378-385.

5. Scarsi K, Lamorde M, Darin K, et al. Efavirenz- but not nevirapine-based antiretroviral therapy decreases exposure to the levonorgestrel released from a sub-dermal contraceptive implant. J Int AIDS Soc. 2014;17:19484.

6. Patel RC, Onono M, Gandhi M, et al, Pregnancy rates in HIV-positive women using contraceptives and efavirenz-based or nevirapine-based antiretroviral therapy in Kenya: a retrospective cohort study. Lancet $H I V .2015 ; 2: \mathrm{e} 474-\mathrm{e} 482$.

7. Scarsi KK, Darin KM, Nakalema S, et al. Unintended pregnancies observed with combined use of the levonorgestrel contraceptive implant and efavirenz-based antiretroviral therapy: a three-arm pharmacokinetic evaluation over 48 weeks. Clin Infect Dis. 2016;62:675-682.

8. Landolt NK, Phanuphak N, Ubolyam S, et al., Significant Decrease of ethinylestradiol with nevirapine, and of etonogestrel with efavirenz in HIV-positive women. J Acquir Immune Defic Syndr. 2014;66:E50-E52.

9. Perry SH, Swamy P, Preidis GA, et al. Implementing the Jadelle implant for women living with HIV in a resource-limited setting: concerns for drug interactions leading to unintended pregnancies. AIDS. 2014;28:791-793.

10. Nel A, van Niekerk N, Kapiga S, et al. Safety and efficacy of a dapivirine vaginal ring for HIV prevention in women. $N$ Engl $J$ Med. 2016;375: 2133-2143.

11. Baeten JM, Palanee-Phillips T, Brown ER, et al. Use of a vaginal ring containing dapivirine for HIV-1 prevention in women. $N$ Engl $J$ Med. 2016:;375:2121-2132.

12. Brown ER, Palanee-Philips T, Marzinke MA, et al. Residual dapivirine ring levels indicate higher adherence to vaginal ring is associated with HIV-1 protection (TUACO 105LB). 21st International AIDS Conference, 18-22 July 2016.

13. Makanani B, Balkus JE, Palanee-Philips T, et al. Pregnancy incidence and outcomes among women using the dapivirine vaginal ring. Conference on Retroviruses and Opportunistic Infections 2016; Seattle, WA.

14. Seserko LA, Emory JF, Hendrix CW, et al. The development and validation of an UHPLC-MS/MS method for the rapid quantification of the antiretroviral agent dapivirine in human plasma. Bioanalysis. 2013;5:2771-2783.

15. Trussell J. Contraceptive failure in the United States. Contraception. 2011;83:397-404

16. Hanley JA, Lippman-Hand A. If nothing goes wrong, is everything all right -interpreting zero numerators. JAMA. 1983;249:1743-1745.

17. Rothman K, Boice J. Epidemiologic Analysis With a Programmable Calculator. Washington, DC: U.S. Department of Health, Education and Welfare; 1979.

18. Andersen PK, Gill RD. Cox's regression model for counting processes: a large sample study. Ann Stat. 1982;10:1100-1120.

19. To EE, Hendrix CW, Bumpus NN. Dissimilarities in the metabolism of antiretroviral drugs used in HIV pre-exposure prophylaxis in colon and vagina tissues. Biochem Pharmacol. 2013;86:979-990.

20. Nel A, Smythe S, Young K, et al. Safety and pharmacokinetics of dapivirine delivery from matrix and reservoir intravaginal rings to HIVnegative women. J Acquir Immune Defic Syndr. 2009;51:416-423.

21. Romano J, Variano B, Coplan P, et al. Safety and availability of dapivirine (TMC120) delivered from an intravaginal ring. AIDS Res Hum Retroviruses. 2009;25:483-488.

22. Van Damme L, Corneli A, Ahmed K, et al. Preexposure prophylaxis for HIV infection among African women. N Engl J Med. 2012;367:411-422.

23. Balkus JE, Brown ER, Hillier SL, et al. Oral and injectable contraceptive use and HIV acquisition risk among women in four African countries: a secondary analysis of data from a microbicide trial. Contraception. 2016;93:25-31.

24. Murnane PM, Heffron R, Ronald A, et al. Pre-exposure prophylaxis for HIV-1 prevention does not diminish the pregnancy prevention effectiveness of hormonal contraception. AIDS. 2014;28:1825-1830.

25. Nanda K, Callahan R, Taylor D, et al. Medroxyprogesterone acetate levels among Kenyan women using depot medroxyprogesterone acetate in the FEM-PrEP trial. Contraception. 2016;94:40-47.

26. Pyra M, Heffron R, Mugo NR, et al. Effectiveness of hormonal contraception in HIV-infected women using antiretroviral therapy. AIDS. 2015;29: 2353-2359. 